\title{
A FULLY PARALLEL METHOD FOR TRIDIAGONAL EIGENVALUE PROBLEM
}

\author{
KUIYUAN LI \\ Department of Mathematics and Statistics \\ University of West Florida \\ Pensacola, FL 32514 \\ (Received July 23, 1992)
}

ABSTRACT. In this paper, a fully parallel method for finding all eigenvalues of a real matrix pencil $(A, B)$ is given, where $A$ and $B$ are real symmetric tridiagonal and $B$ is positive definite. The method is based on the homotopy continuation coupled with the strategy 'Divide-Conquer' and Laguerre iterations. The numerical results obtained from implementation of this method on both single and multiprocessor computers are presented. It appears that our method is strongly competitive with other methods. The natural parallelism of our algorithm makes it an excellent candidate for a variety of advanced architectures.

KEY WORDS AND PHRASES. Eigenvalues, Eigenvalue curves, Multiprocessors, Homotopy method.

1992 AMS SUBJECT CLASSIFICATION CODE. 65F15.

1. INTRODUCTION.

When $B$ is a well-conditioned positive definite matrix, real symmetric generalized eigenvalue problem

$$
A x=\lambda B x
$$

can be reduced to the form

$$
\mathrm{L}^{-1} A L^{-T}\left(L^{T} x\right)=\lambda\left(L^{T} x\right)
$$

where $A$ and $B$ are real $n \times n$ symmetric matrices and $B=L L^{T}$. There are many very efficient algorithms for (2), for instant, the QR algorithm [8], the D\&C algorith [3], the bisection algorithm [5] and the homotopy algorithm [6]. When $A$ and $B$ are both tridiagonal the above technique is unattractive because $L^{-1} A L^{-T}$ is, in general, a full matrix.

In this paper, we shall present a parallel homotopy method for finding all the eigenvalues or all eigenpairs of a matrix pencil $(A, B)$, where $A$ and $B$ are both real symmetric tridiagonal and $B$ is positive definite. Assume in (1),

$$
A=\left(\begin{array}{ccccc}
\alpha_{1} & \beta_{2} & & & \\
\beta_{2} & \alpha_{2} & \beta_{3} & & \\
& \ddots & \ddots & \ddots & \\
& & \beta_{n-1} \alpha_{n-1} & \beta_{n} \\
& & & \beta_{n} & \alpha_{n}
\end{array}\right) \text { and } B=\left(\begin{array}{ccccc}
\delta_{1} & \gamma_{2} & & & \\
\gamma_{2} & \delta_{2} & \gamma_{3} & \\
& \ddots & \ddots & \ddots & \\
& & \gamma_{n-1} & \delta_{n-1} \gamma_{n} \\
& & & \gamma_{n} & \delta_{n}
\end{array}\right)
$$


If $\beta_{i}=\gamma_{i}=0$ for some $i$, then (1) can clearly be decomposed into two subproblems and we can solve them independently. Hence, we will assume all $\beta_{i}$ and $\gamma_{i}$ are not both equal to zero.

Let

$$
C=\left(\begin{array}{cc}
A_{1} & 0 \\
0 & A_{2}
\end{array}\right)
$$

where

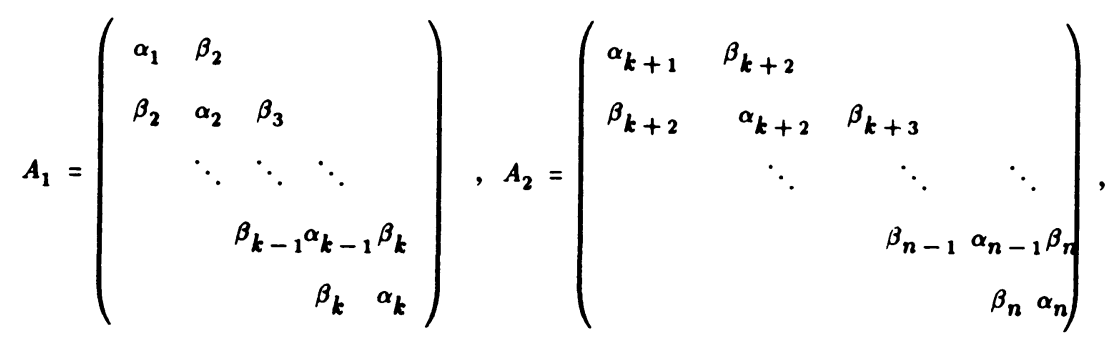

$$
\begin{aligned}
& D=\left(\begin{array}{cc}
B_{1} & 0 \\
0 & B_{2}
\end{array}\right) \text {, }
\end{aligned}
$$

where

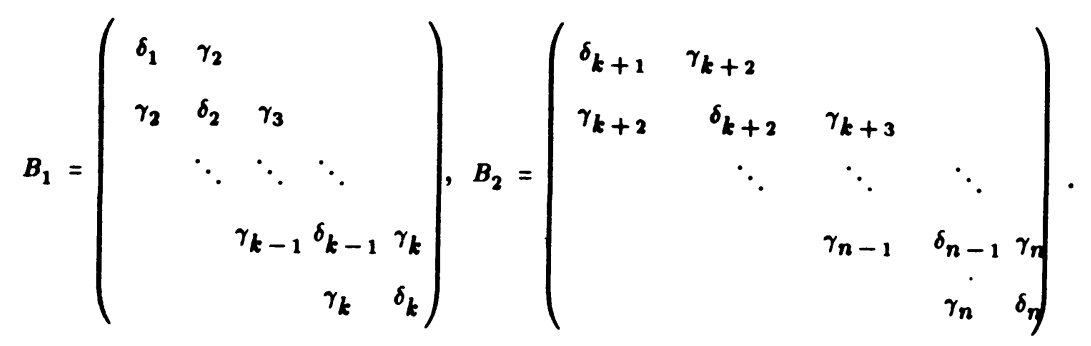

Consider the homotopy $H: R \times[0,1] \rightarrow R$, defined by

$$
\begin{aligned}
H(\lambda, t) & =\operatorname{det}((1-t)(C-\lambda D)+t(A-\lambda B)) \\
& =\operatorname{det}(A(t)-\lambda B(t)),
\end{aligned}
$$

where $A(t)=(1-t) C+t A$ and $B(t)=(1-t) D+t B$. The pencil $(C, D)$ is called an initial pencil .

In section 2, we shall show that the solution set of $H(\lambda, t)=0$ in (6) consists of continuously differentiable curves $\lambda(t)$, each joins an eigenvalue of $(C, D)$ to one of $(A, B)$. We call each of these curves a homotopy curve or an eigencurve. We shall also show that each eigenvalue curve is monotonic in $t$. And if $m$, the multiplicity of an eigencurve $\lambda(t)$ is greater than one in any subinterval of $[0,1]$, then it must be a constant curve. In the consequence, it is an eigenvalue of $(A, B)$ of multiplicity of $m$ or $m+1$. We shall give the details of our algorithm in section 3 and some numerical results will be presented in section 4 .

2. PRELIMINARY ANALYSIS

Proposition 2.1. Let $H(\lambda, t)$ be defined as in (6), then the solution set of $H(\lambda, t)=0$ consists of real, continuously differentiable eigencurves.

PRO0F. First of all, we show that $\lambda(t)$, a solution of $H(\lambda(t), t)=0$ is real for any $t$ in $[0,1]$. Since $H(\lambda(t), t)=\operatorname{det}(A(t)-\lambda(t) B(t))$, we only need to show that $B(t)$ is positive definite for all $t$ in $[0,1]$. Let $\lambda_{1}(t)$ be the smallest eigenvalue 
of $B(t)$, then by Cauchy's interlace theorem [8], $\lambda_{1}(0)>\lambda_{1}(t)$ for all $t$ in $(0,1]$. By Proposition 2.1 in [6], $\lambda_{1}(t)$ is strictly monotonic in $t$. Therefore, $\lambda_{1}(t)$ is strictly monotone decreasing in $t$. Hence $\lambda_{1}(t)>\lambda_{1}(1)>0$ for all $t$ in $[0,1)$ since $B(1)=B$ is positive definite. Hence $B(t)$ is positive definite for all $t$ in $[0,1]$.

Now we show that $\lambda(t)$ is continuously differentiable. Clearly, $H(\lambda, t)$ can be written as:

$$
H(\lambda(t), t)=c_{n}(t) \lambda^{n}(t)+c_{n-1}(t) \lambda^{n-1}(t)+\cdots+c_{1}(t) \lambda(t)+c_{0}(t)
$$

where $c_{i}(t)$ 's are polynomials in $t$. Let $\lambda(t)$ be a solution of $H(\lambda(t), t)=0$ and $t_{0}$ any point in $(0,1)$. By Puiseux's theorem [10],

$$
\lambda\left(t_{0}+\epsilon\right)=\lambda\left(t_{0}\right)+b_{1} \epsilon^{\frac{1}{h}}+b_{2} \epsilon^{\frac{2}{h}}+\cdots
$$

converges for sufficiently small $\epsilon$. Let $b_{m}$ denote the first nonzero coefficient, then

$$
b_{m}=\lim _{\epsilon \rightarrow 0+} \frac{\lambda\left(t_{0}+\epsilon\right)-\lambda\left(t_{0}\right)}{\epsilon^{\frac{m}{h}}}
$$

is real since $\lambda\left(t_{0}+\epsilon\right), \lambda\left(t_{0}\right)$ and $\epsilon$ are all real. On the other hand,

$$
(-1)^{\frac{m}{h}} b_{m}=\lim _{\epsilon \rightarrow 0-} \frac{\lambda\left(t_{0}+\epsilon\right)-\lambda\left(t_{0}\right)}{(-\epsilon)^{\frac{m}{h}}}
$$

is also real. Hence, $(-1)^{\frac{m}{h}}$ is a real number. Therefore, $m$ must be a multiple of $h$. We can continue the same argument to show that only integral powers of $c$ can have nonzero coefficients. Therefore, $\lambda\left(t_{0}+\epsilon\right)=\sum_{i=0}^{\infty} b_{i} \epsilon^{i}$. Hence, $\lambda(t)$ is continuously differentiable.

Q.E.D.

PROPOSITION 2.2. Any eigencurve $\lambda(t)$ of $(A(t), B(t))$ is either straight line or strictly monotonic in $t$.

PROOF: Since

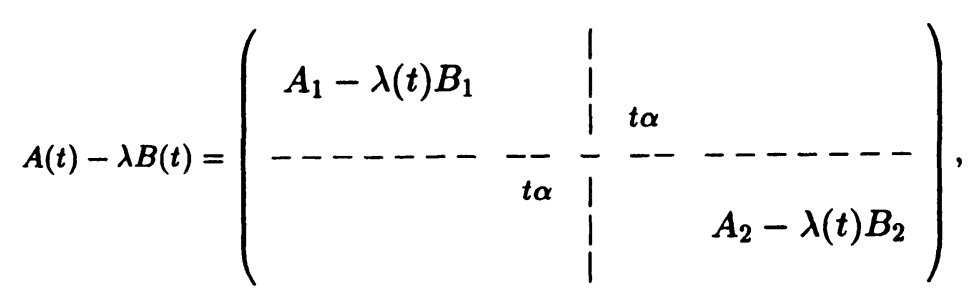

where $\alpha=\beta_{k+1}-\lambda(t) \gamma_{k+1}$, there exist polynomials $f_{1}(\lambda)$ and $f_{2}(\lambda)$ such that $H(\lambda, t)=f_{1}(\lambda)-t^{2} f_{2}(\lambda)$. If there exists a $\lambda_{0}$ such that $f_{2}\left(\lambda_{0}\right)=0$, then $H\left(\lambda_{0}, t\right)=0$ implies $f_{1}\left(\lambda_{0}\right)=0$. Hence $H\left(\lambda_{0}, t\right)=0$ for all $t$ in $[0,1]$. Therefore, $\lambda(t)=\lambda_{0}$ for all $t$ in $[0,1]$, i.e., $\lambda(t)$ is a straight line.

If for any $\lambda, f_{2}(\lambda) \neq 0$, then $H\left(\lambda_{0}, t\right)=0$ has at most one solution in $[0,1]$. Therefore, $\lambda(t)$ must be strictly monotonic in $t$. Otherwise, for certain $\lambda_{0}$, $H\left(\lambda_{0}, t\right)=0$ will have more than one solution in $[0,1]$ (See Figure 1 ). 


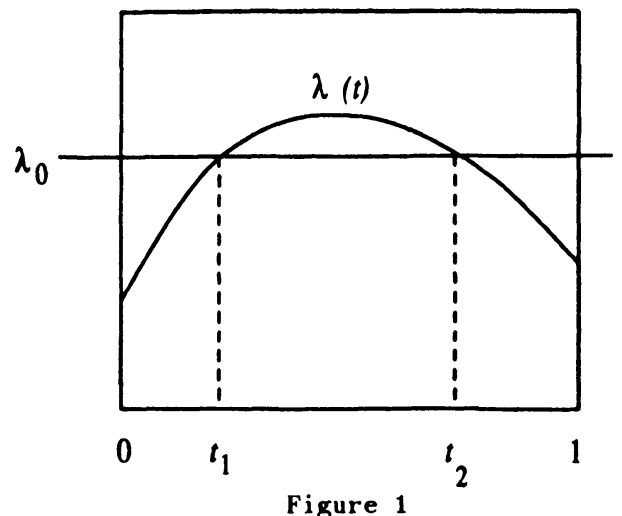

Let $m(\lambda(t))$ denote the multiplicity of $\lambda(t)$. Proposition 2.2 implies that if $m(\lambda(t))=1$ and there are $t_{1}$ and $t_{2}$ in $[0,1]$ such that $\lambda\left(t_{1}\right)=\lambda\left(t_{2}\right)$, where $t_{1} \neq t_{2}$, then $\lambda(t)$ must be constant.

PROPOSITION 2.3. Let $\lambda(t)$ be an eigencurve. If $[a, b]$ is a subinterval of $[0,1]$ and $m(\lambda(t))=m>1$, for any $t$ in $[a, b]$, then $\lambda(t) \equiv \lambda(0)$ and $\lambda(0)$ is an ezgenvalue of matrix pencil $(A, B)$ of multiplicity of $m$ or $m$ +1 .

PROOF. Claim 1: $\lambda(t) \equiv \lambda_{0}$ for some constant $\lambda_{0}$, for $t$ in $[a, b]$.

Let $t_{1}, t_{2}, \cdots, t_{n}$ be $n$ distinct points in, $[a, b]$. Since rank $(A(t)-\lambda(t) B(t))=n-m<n-1$ for any $t$ in $[a, b]$ and $A(t)-\lambda(t) B(t)$ is symmetric tridiagonal, at least one of the off-diagonal elements of $A(t)-\lambda(t) B(t)$, say $\beta_{s}-\lambda(t) \gamma_{s}$, is equal to zero at two points, say $t_{i}$ and $t_{j} \cdot$ Otherwise, rank $(A(t)-\lambda(t) B(t)) \geq n-1$. Hence, $\beta_{s}-\lambda\left(t_{i}\right) \gamma_{s}=0$ and $\beta_{s}-\lambda\left(t_{j}\right) \gamma_{s}=0$. Since $\beta_{s}$ and $\gamma_{s}$ are not both equal to zero, $\lambda\left(t_{i}\right)=\lambda\left(t_{j}\right)$. Hence, $\lambda(t) \equiv \lambda_{0}$ for some constant $\lambda_{0}$, for any $t$ in $[a, b]$ by Proposition 2.2.

Claim 2: $\operatorname{rank}\left(A(t)-\lambda_{0} B(t)\right)=n-m$ for all $t$ in $(0,1]$ except at possible one point in $(0,1] \operatorname{rank}\left(A(t)-\lambda_{0} B(t)\right)=n-m-1$.

If $\beta_{k+1}-\lambda_{0} \gamma_{k+1}=0$, then clearly, $\operatorname{rank}\left(A(t)-\lambda_{0} B(t)\right)=n-m$ for all, $t$ in $(0,1]$. If $\beta_{k+1}-\lambda_{0} \gamma_{k+1} \neq 0$, since some off diagonal elements of $A(t)-\lambda_{0} B(t)$ are equal to zero, $A(t)-\lambda_{0} B(t)$ can be rewritten as:

$$
A(t)-\lambda_{0} B(t)=\left(\begin{array}{lll}
A_{1}-\lambda_{0} B_{1} & & \\
& A_{2}(t)-\lambda_{0} B_{2}(t) & \\
& & A_{3}-\lambda_{0} B_{3}
\end{array}\right)
$$

where $A_{2}(t)-\lambda_{0} B_{2}(t)$ is unreduced symmetric tridiagonal, i.e., all its off-diagonal elements are not equal to zero.

Hence,

$$
\begin{aligned}
\operatorname{rank}\left(A(t)-\lambda_{0} B(t)\right) & =\operatorname{rank}\left(A_{1}-\lambda_{0} B_{1}\right)+\operatorname{rank}\left(A_{2}(t)-\lambda_{0} B_{2}(t)\right) . \\
& +\operatorname{rank}\left(A_{3}-\lambda_{0} B_{3}\right)
\end{aligned}
$$

Clearly, $\operatorname{rank}\left(A_{1}-\lambda_{0} B_{1}\right)+\operatorname{rank}\left(A_{3}-\lambda_{0} B_{3}\right)$ is constant. Assume $\operatorname{rank}\left(A_{2}(t)-\lambda_{0} B_{2}(t)\right)=m_{0}$ for some integer $m_{0}$ for all $t$ in $[a, b]$. Since $\left(A_{2}(t)-\lambda_{0} B_{2}(t)\right)$ is a unreduced symmetric tridiagonal matrix, its eigencurves are smooth, disjoint and monotonic in $t$ by Proposition 2.1. in [6]. Therefore, if $\mu(t)$ is an eigencurve of 
$\left(A_{2}(t)-\lambda_{0} B_{2}(t)\right)$, i.e., $\operatorname{det}\left(\left(A_{2}(t)-\lambda_{0} B_{2}(t)\right)-\mu(t)\right)=0$, then either $\mu(t) \equiv 0$ or there is at most one $t$ such that $\mu(t)=0$. Hence, if zero is an eigenvalue of $\left(A_{2}(t)\right.$ $\left.-\lambda_{0} B_{2}(t)\right)$ for some $t$ in $[a, b]$, then $\mu(t)=0$ is an eigenvalue for any $t$ in $[0,1]$ since $\operatorname{rank}\left(A_{2}(t)-\lambda_{0} B_{2}(t)\right)=m_{0}$ for all $t$ in $[a, b]$. Therefore, $\operatorname{rank}\left(A_{2}(t)-\lambda_{0} B_{2}(t)\right)=m_{0}$ for all $t$ in $(0,1]$. If for any $t$ in $(0,1]$, zero is not an eigenvalue of $\left(A_{2}(t)-\lambda_{0} B_{2}(t)\right)$, then $\operatorname{rank}\left(A_{2}(t)-\lambda_{0} B_{2}(t)\right)=m_{0}$. Hence, $\operatorname{rank}\left(A_{2}(t)-\lambda_{0} B_{2}(t)\right)=n-m$ for all $t$ in $(0,1]$ in these two cases. If for any $t$ in $[a, b]$, zero is not an eigenvalue of $\left(A_{2}(t)-\lambda_{0} B_{2}(t)\right)$, then clearly, there is at most one $t$ in $(0,1]$ such that $\operatorname{rank}\left(A_{2}(t)-\lambda_{0} B_{2}(t)\right)=m_{0}-1$.

Hence, $\lambda(t)$ is constant for all $t$ in $[0,1]$ and $\lambda(0)$ is an eigenvalue of $(A, B)$ of multiplicity of $m$ or $m+1$.

Q.E.D.

From Proposition 2.3, we will not have any those bifurcation curves in Figure 2 .

If $\lambda(t)$ is not constant, then its multiplicity must be 1 .

Let $\lambda_{1}(t), \lambda_{2}(t), \ldots, \lambda_{n}(t)$ be $n$ eigencurves of $(A(t), B(t))$, where $\lambda_{1}(0) \leq \lambda_{2}(0)$ $\leq, \cdots, \leq \lambda_{n}(0)$.

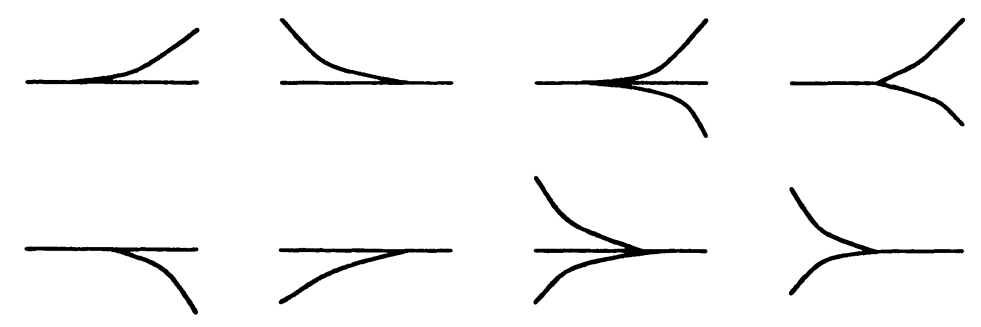

Figure 2

PROPOSITION 2.4. Let $\lambda_{i}(t)$ and $\lambda_{i+1}(t)$ be two nonconstant eigencurves of $(A(t), B(t))$. If $\lambda_{i}\left(t_{0}\right)$ $<\lambda_{i+1}(t)$ for some $t_{0}$ in $(0,1]$, then $\lambda_{i}(t)<\lambda_{i+1}(t)$ for all $t$ in $(0,1]$.

PROOF. From Proposition 2.2 and 2.3 , both $\lambda_{i}(t)$ and $\lambda_{i+1}(t)$ must be strictly monotonic in $t$. Assume $\lambda_{i}(t)$ and $\lambda_{i+1}(t)$ are both strictly monotone increasing. If there exists a $t_{1}$ in $(0,1]$ such that $\lambda_{i}\left(t_{1}\right)$ and $\lambda_{i+1}\left(t_{1}\right)$, then $\lambda_{i}\left(t_{1}\right)$ and $\lambda_{i+1}(0)$ since $\lambda_{i+1}(t)$ is strictly monotonic in $t$. Therefore, $t_{2}>0$ is a solution of $H\left(\lambda_{i+1}(0), t\right)=0$ (See Figure 3). By Proposition 2.2, $\lambda_{i+1}(t)$ is a constant. Contradiction.

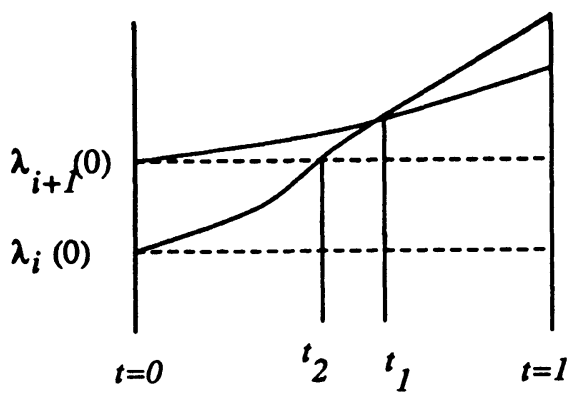

Figure 3

Similarly, if $\lambda_{i}(t)$ and $\lambda_{i+1}(t)$ are both strictly monotone decreasing, then the conclusion holds. 
Assume $\lambda_{i}(t)$ is monotone increasing and $\lambda_{i+1}(t)$ monotone decreasing, then $\lambda_{i}(0)<\lambda_{i+1}(0)$. If there is a $t_{1}>0$ such that $\lambda_{i}\left(t_{1}\right)$ and $\lambda_{i+1}\left(t_{1}\right)$, then there exists a $t_{2}>t_{1}$ such that $\lambda_{i+1}\left(t_{1}\right)<\lambda_{i}\left(t_{2}\right)<\lambda_{i+1}(0)$. Since $\lambda_{i+1}(t)$ is continuous and $\lambda_{i+1}\left(t_{1}\right)<\lambda_{i+1}(t)<\lambda_{i+1}(0)$ for any $t$ in $\left(0, t_{1}\right)$, there is a $t_{3}$ in $\left(0, t_{1}\right)$ such that $\lambda_{i+1}\left(t_{3}\right)=\lambda\left(t_{2}\right)$ (See Figure 4), then $H\left(\lambda_{i}\left(t_{2}\right), t\right)=0$ has two solutions. Therefore, either $\lambda_{i}(t)$ or $\lambda_{i+1}(t)$ must be constant. Contradiction.

Clearly, if $\lambda_{i+1}(t)$ is increasing and $\lambda_{i}(t)$ decreasing, then the conclusion holds since $\lambda_{i}(0)<\lambda_{i+1}(0)$.

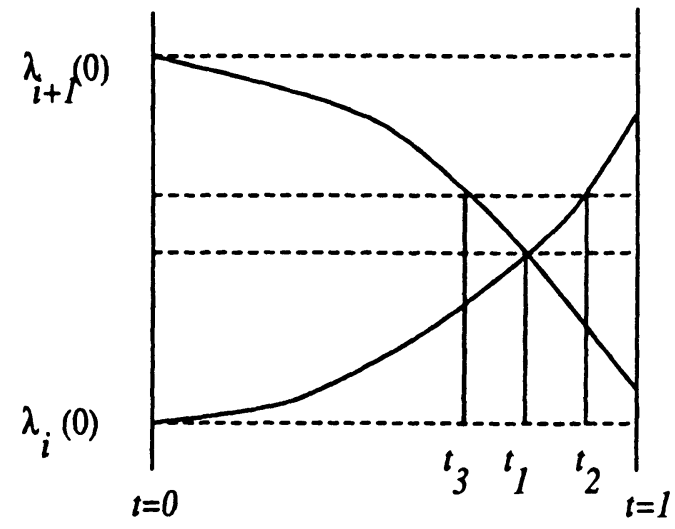

Q.E.D.

Figure 4

From Proposition 2.4, if $\lambda_{i}(t)$ and $\lambda_{i+1}(t)$ are both constants or both are not constants, then they must be disjoint. However, if one of them is constant, they may cross.

Example 1.

$$
A=\left(\begin{array}{lll}
4 & 1 & 0 \\
1 & 1 & 4 \\
0 & 4 & 1
\end{array}\right), \quad B=\left(\begin{array}{lll}
4 & 1 & 0 \\
1 & 3 & 0 \\
0 & 0 & 3
\end{array}\right)
$$

Let

$$
A(t)=\left(\begin{array}{ccc}
4 & 1 & 0 \\
1 & 1 & 4 t \\
0 & 4 t & 1
\end{array}\right), \quad B(t)=\left(\begin{array}{lll}
4 & 1 & 0 \\
1 & 3 & 0 \\
0 & 0 & 3
\end{array}\right)
$$

then $(A(t), B(t))$ has three eigencurves:

$$
\begin{aligned}
& \lambda_{1}(t)=\frac{20-\sqrt{4+8448 t^{2}}}{66}, \\
& \lambda_{2}(t)=\frac{20+\sqrt{4+8448 t^{2}}}{66},
\end{aligned}
$$

and

$$
\lambda_{3}=1
$$

From these propositions, we know that all eigenvalues of the initial pencil not only close to the eigenvalues of pencil $(A, B)$, but also separate them. These results provide us very important information in designing our code. (See Figure 5). 


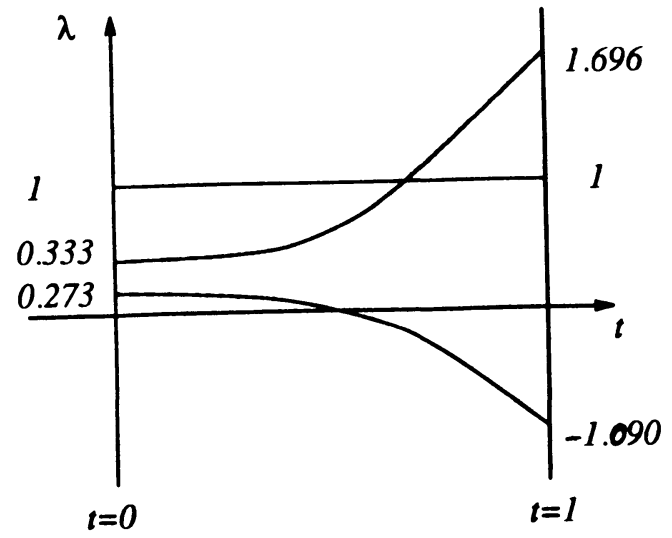

Figure 5

\section{ALGORITHM}

Our algorithm is based on following steps.

(i) Form initial matrix pencil.

For a given matrix pencils $(A, B)$, let $k=[n / 2]$, then form an initial matrix pencil as in (4) and (5) in Section 1 . If $k$ is greater than 2 , repeat above procedure on those submatrices, until the dimension of each submatrices is less than or equal to 2 . Now, $(C, D)$ is the initial matrix pencil, where $C=\operatorname{drag}\left(A_{s, 1}\right.$, $\left.A_{s, 2}, \ldots, A_{s, p}\right)$ and $D=\operatorname{diag}\left(B_{s, 1}, B_{s, 2}, \ldots, B_{s, p}\right), p=2^{0}$ for some positive integer $s$. We have a tree (See Figure 6).

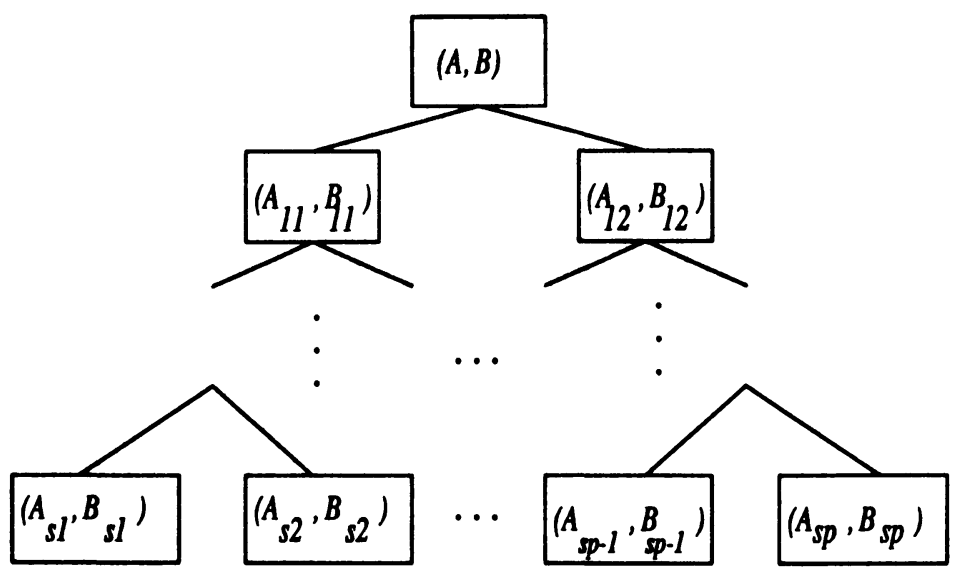

Figure 6

(ii) Compute all eigenvalues of $(C, D)$.

Compute the eigenvalues of each submatrix pencil $\left(A_{s, i}, B_{s, i}\right), i=1,2, \ldots, p$. Since $A_{s i}$ and $B_{s i}$ are at most $2 \times 2$ matrices, the eigenvalues of pencil $\left(A_{s i}, B_{s i}\right)$ can be easily obtained.

(iii) Conquer.

After all the eigenvalues of each submatrix pencil $\left(A_{s i}, B_{s i}\right) i=1,2, \ldots, p$ are available, we then compute all the eigenvalues of each submatrix pencil ( $A_{s i}$, $\left.B_{s i}\right), i=1,2, \ldots, p / 2$ by simple step homotopy method with Laguerre iterations.

It is sufficient to assume that all the eigenvalues of matrix pencils ( $A_{11}$, $\left.B_{11}\right)$ and $\left(A_{12}, B_{12}\right)$ are available and we want to compute all eigenvalues of matrix pencil $(A, B)$. 
Let

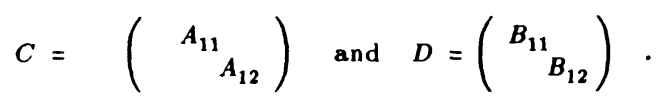

Construct a homotopy as in (6):

$$
\begin{aligned}
H(\lambda, t) & =\operatorname{det}((1-t)(C-\lambda D)+t(A-\lambda B)) \\
& =\operatorname{det}(A(t)-\lambda B(t)),
\end{aligned}
$$

where $A(t)=(1-t) C+t A$ and $B(t)=(1-t) D+t B$.

Let $\lambda_{1}(t), \quad \lambda_{2}(t), \ldots, \lambda_{n}(t)$ be $n$ eigencurves of $H(\lambda, t)$, where $\lambda_{1}(0) \leq \lambda_{2}(0) \leq, \ldots, \leq \lambda_{n}(0)$ are the eigenvalues of matrix pencil $(C, D)$. We conquer $(C, D)$ to $(A, B)$ with Laguerre iteration [12], i.e., use the eigenvalues of $(C, D)$ as starting points of Laguerre iterations to compute all eigenvalues of $(A, B)$.

(a) Some knowledge of the Laguerre iteration.

Let $f(\lambda)$ be a function having $n$ real zeros $\lambda_{1}<\lambda_{2}<, \ldots,<\lambda_{n}$ and $x_{0}$ lie between $\lambda_{i}$ and $\lambda_{i+1}$, then Laguerre iteration gives the two sequences

$$
x_{m+1}=x_{m}-\frac{n f\left(x_{m}\right)}{f^{\prime}\left(x_{m}\right) \pm \sqrt{(n-1)^{2}\left(f^{\prime}\left(x_{m}\right)\right)^{2}-n(n-1) f\left(x_{m}\right) f^{\prime \prime}\left(x_{m}\right)}} .
$$

One of which converges to $\lambda_{i}$, another to $\lambda_{i+1}$ monotonicly. In the neighborhood of a simple zero at $\lambda_{i}$, (7) converges cubicly to $\lambda_{i}$. On the other hand, in the neighborhood of a zero $\lambda_{i}$ of multiplicity $r$,

$$
x_{m+1}=x_{m}-\frac{n f\left(x_{m}\right)}{f^{\prime}\left(x_{m}\right)+\operatorname{sign}\left(f^{\prime}\left(x_{m}\right)\right) \sqrt{\frac{(n-r)}{r}\left(\left(f^{\prime}\left(x_{m}\right)\right)^{2}-n f\left(x_{m}\right) f^{\prime \prime}\left(x_{m}\right)\right)}}
$$

also converges to $\lambda_{i}$ cubicly.

(b) Computing $f(\lambda), f^{\prime}(\lambda)$ and $f^{\prime \prime}(\lambda)$.

Let $f(\lambda)=\operatorname{det}(A-\lambda B)$, then all the zeros of $f(\lambda)$ are real. Since

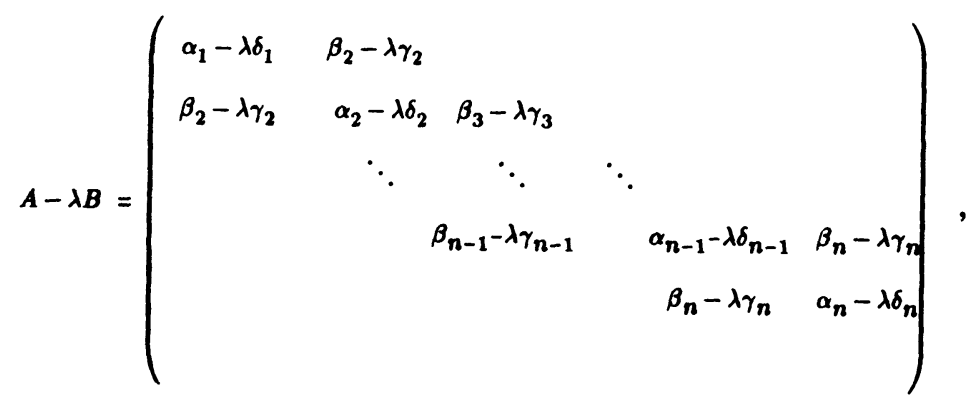

we have the following three ter recursion.

$$
\begin{aligned}
& p_{0}(\lambda)=1 \\
& p_{1}(\lambda)=\left(\alpha_{1}-\lambda \delta_{1}\right) \\
& p_{m}(\lambda)=\left(\alpha_{m}-\lambda \delta_{m}\right) p_{m-1}(\lambda)-\left(\beta_{m}-\lambda \gamma_{m}\right)^{2} p_{m-2}(\lambda), m=2, \ldots, n \\
& f(\lambda)=p_{n}(\lambda) .
\end{aligned}
$$

By simple computation, we have following:

$$
\begin{aligned}
& p_{0}^{\prime}(\lambda)=0 \\
& p_{1}^{\prime}(\lambda)=-\delta_{1}
\end{aligned}
$$




$$
\begin{aligned}
p_{m}^{\prime}(\lambda)= & \left(\alpha_{m}-\lambda \delta_{m}\right) p_{m-1}^{\prime}(\lambda)-\delta_{m} p_{m-1}(\lambda) \\
& -\left(\beta_{m}-\lambda \gamma_{m}\right)^{2} p_{m-2}^{\prime}(\lambda)+2\left(\beta_{m}-\lambda \gamma_{m}\right) \gamma_{m} p_{m-2}(\lambda) \\
& m=2,3, \ldots, n \\
f^{\prime}(\lambda)= & p_{n}^{\prime}(\lambda)
\end{aligned}
$$

and

$$
\begin{aligned}
& p_{0}^{\prime \prime}(\lambda)=0 \\
& p_{1}^{\prime \prime}(\lambda)=0 \\
& p_{m}^{\prime \prime}(\lambda)=\left(\alpha_{m}-\lambda \delta_{m}\right) p_{m-1}^{\prime \prime}(\lambda)-\left(\beta_{m}-\lambda \gamma_{m}\right)^{2} p_{m-2}^{\prime \prime}(\lambda)-2 \delta_{m} p_{m-1}^{\prime}(\lambda) \\
& +4\left(\beta_{m}-\lambda \gamma_{m}\right) \gamma_{m} p_{m-2}^{\prime}(\lambda)-2 \gamma_{m}^{2} p_{m-2}(\lambda) \\
& m=2,3, \ldots, n \\
& f^{\prime \prime}(\lambda)=p_{n}^{\prime \prime}(\lambda)
\end{aligned}
$$

By computing $p_{m}(\lambda), m=0,1,2, \ldots, n$, we know exactly how many eigenvalues of $(A, B)$ are less than $\lambda$. Since $x_{m}$ converges monotonicly, after one Laguerre iteration, we know exactly $x_{m}$ converges to which eigenvalue of $(A, B)$ without any extra computations.

(c) The overflow and the underflow control.

Since $f(\lambda)=\operatorname{det}(A-\lambda B), f(\lambda)$ could be very small or large. Since $f(\lambda)$ can be written as

$$
\begin{aligned}
& f(\lambda)=\Pi_{i=1}^{n} a_{i}\left(\lambda-\lambda_{i}\right), \\
& f^{\prime}\left(\lambda=\sum_{j=1}^{n} \Pi_{i \neq j}^{n} a_{i}\left(\lambda-\lambda_{i}\right)\right.
\end{aligned}
$$

and

$$
f^{\prime \prime}(\lambda)=\sum_{k=1}^{n} \sum_{j=1}^{n} \Pi_{i \neq j, k}^{n} a_{i}\left(\lambda-\lambda_{i}\right) .
$$

We can see that we can find $\beta$ such that $f(\lambda)=\alpha 10^{\beta}, f^{\prime}(\lambda)=\gamma 10^{\beta}$ and $f^{\prime \prime}(\lambda)=$ $\delta 10^{\beta}$, where $\alpha, \gamma$ and $\delta$ have magnitude between the machine minimum and maximum numbers. Hence, when we compute $p_{m}(\lambda), p_{m}^{\prime}(\lambda)$ and $p_{m}^{\prime \prime}(\lambda)$, if $p_{m}(\lambda)$ is too large (or too smal1), then we multiply $p_{m}(\lambda), p_{m}^{\prime}(\lambda)$ and $p_{m}^{\prime \prime}(\lambda)$ by $10^{\beta}$ for some integer $\beta$ so that $p_{m}(\lambda) 10^{\beta}$ is not too large (or too small). In this way, we can avoid overflow as well as underflow. Finally, we will get $\tilde{p}_{n}, \tilde{p}_{n}^{\prime}(\lambda)$ and $\tilde{p}_{n}^{\prime \prime}(\lambda)$ and all of them have magnitude between machine minimum and maximum numbers and $f(\lambda)$ $=10^{s} \tilde{p}_{n}, f^{\prime}(\lambda)=10^{8} \widetilde{p}_{n}^{\prime}$ and $f^{\prime \prime}(\lambda)=10^{8} \tilde{p}_{n}^{\prime \prime}$, for some integer s. Since

$$
\begin{aligned}
& x_{m+1}=x_{m}-\frac{n f\left(x_{m}\right)}{f^{\prime}\left(x_{m}\right) \pm \sqrt{(n-1)^{2}\left(f^{\prime}\left(x_{m}\right)\right)^{2}-n(n-1) f\left(x_{m}\right) f^{\prime \prime}\left(x_{m}\right)}} \\
& =x_{m}-\frac{n \tilde{p}_{n}\left(x_{m}\right)}{\tilde{p}_{n}^{\prime}\left(x_{m}\right) \pm \sqrt{(n-1)^{2}\left(\tilde{p}_{n}^{\prime}\left(x_{m}\right)\right)^{2}-n(n-1) \tilde{p}\left(x_{m}\right) \tilde{p}_{n}^{\prime \prime}\left(x_{m}\right)}},
\end{aligned}
$$

overflow and underflow can be avoided.

Now we give details of computing eigenvalues of $(A, B)$.

Step 1. If $i \neq 1$, go to step 2. Use $\lambda_{1}(0)$ as a starting point to do Laguerre iteration to locate $\mu_{1}$, the smallest eigenvalue of $(A, B)$. By computing $f\left(\lambda_{1}(0)\right)$, we know exactly if $\left\{x_{m}\right\}$, the sequence generated by Laguerre iterations, will converge to $\mu_{1}$. If it does not, then use $\lambda_{1}(0)-\alpha$ as a starting point, where $\alpha=$ $2\left|\beta_{k+1}\right| \gamma_{k+1} \mid$ if $\gamma_{k+1} \neq 0$ and $\alpha=2\left|\beta_{k+1}\right|$ if $\gamma_{k+1}=0$. If $\lambda_{1}(0)<\mu_{1},\left\{x_{m}\right\}$ will converge to $\mu_{1}$.

Step 2. If $\lambda_{i}(0)$ is not a simple eigenvalue of $(C, D)$, then go to step 3 . If $\left|\lambda_{i}(0)-\mu_{i-1}\right|>\epsilon$, where $\epsilon=$ tolerance* $\max \left\{\left|\lambda_{n}(0)\right|,\left|\lambda_{1}(0)\right|\right\}$, use $\lambda_{i}(0)$ as a starting point. Then, $\left\{x_{m}\right\}$ will either converge to $\mu_{i}$ or $\mu_{i+1}$, since the starting point is either in the neighborhood of $\mu_{i}$ or $\mu_{i+1}$ according to the propositions 2.2 and 
2.4 in section 2 . If it converges to $\mu_{i}$, then set $i=i+1$, go to step 5 . If it converges to $\mu_{i+1}$, then use $\left(\mu_{i+1}+\mu_{i-1}\right) / 2$ as a starting point to do Laguerre iteration and the new sequence $\left\{x_{m}\right\}$ will converge to $\mu_{i}$. Set $i=i+2$, go to step 5 .

Step 3. If the multiplicity $r$ of $\lambda_{i}(0)$ is equal to 2 , go to step 4 . by the propositions in last section, $\lambda_{i}(0)$ is an eigenvalue of $(A, B)$. The generalized Sturm sequences at $\lambda_{i}(0) \pm \epsilon$ are computed on $(A, B)$ to check the multiplicity of $\lambda_{i}(0)$. We may have following cases according to the proposition 2.3 .

(a). $\mu_{i}=\mu_{i+1}=\ldots=\mu_{i}+r-1=\lambda_{i}(0)$. Then set $i=i+r$, go to step 5 .

(b). $\mu_{i+1}=\mu_{i+2}=\ldots=\mu_{i+r-1}=\lambda_{i}(0)$. Then use $\left(\lambda_{i}(0)+\mu_{i-1}\right) / 2$ as a starting point to do Laguerre iterations and $\left\{x_{m}\right\}$ will converge to $\mu_{i}$. Then set $i=i+r$, go to step 5 .

(c). $\mu_{i}=\mu_{i+1}=\ldots=\mu_{i+r-2}=\lambda_{i}(0)$. Then use $\left(\lambda_{i}(0)+\lambda_{i}+r(0)\right) / 2$ as a starting point and $\left\{x_{m}\right\}$ will converge to $\mu_{i+r-1}$. Then set $i=i+r$, go to step 5 .

(d). $\mu_{i+1}=\mu_{i+2}=\ldots=\mu_{i+r-2}=\lambda_{i}(0)$. Then use $\left(\mu_{i+1}+\mu_{i-1}\right) / 2$ as, atarting point to do Laguerre iterations and $\left\{x_{m}\right\}$ will converge to $\mu_{i}$. Then use $\left(\lambda_{i}(0)+\lambda_{i+r}(0)\right) / 2$ as a starting point and $\left\{x_{m}\right\}$ will converge to $\mu_{i+r-1}$. Then set $i=i+r$, go to step 5 .

Step 4. The generalized Sturm sequence at $\lambda_{i}(0) \pm c$ are computed on $(A, B)$ to check if $\lambda_{i}(0)$ is an eigenvalue of $(A, B)$. If it is not a eigenvalue of $(A, B)$, then Two sequences $\left\{x_{m}\right\}$ and $\left\{x_{m}^{\prime}\right\}$ will be generated by Laguerre iterations with $\lambda_{i}(0)$ as a starting point. One of then converges to $\mu_{i}$ and another to $\mu_{i+1}$. Then set $i=i+2$, go to step 5 .

If $\lambda_{i}(0)$ is an eigenvalue of $(A, B)$, then we may have following cases according to the proposition 2.2 .

(a). $\mu_{i}=\mu_{i+1}=\lambda_{i}(0)$.

Then set $i=i+2$, go to step 5 .

(b). $\mu_{i+1}=\lambda_{i}(0)$.

Then use $\left(\mu_{i+1}+\mu_{i-1}\right) / 2$ as a starting point to do Laguerre iterations. $\left\{x_{m}\right\}$, generated by Laguerre iteration, will converge to $\mu_{i}$. Then set $i=i+2$, go to step 5.

(c). $\mu_{i}=\lambda_{i}(0)$ and $\mu_{i+1} \neq \lambda_{i}(0)$.

Use $\left(\lambda_{i+2}(0)+\mu_{i}\right) / 2$ as a starting point to do Laguerre iterations. $\left\{x_{m}\right\}$, generated by Laguerre iteration, will converge to $\mu_{i+1}$. Then set $i=i+2$, go to step 5 .

Step 5. If $i>n$, go to (vi). Otherwise, go to step 2 .

(vi) Compute eigenvectors.

If the eigenvectors are required, compute them by inverse iteration.

\section{NUMERICAL RESULTS}

In this section, we present our numerical results. Our homotopy algorith is in its preliminary stage, and much development and testing are necessary. But the numerical results on the examples we have looked at seem remarkable.

EXPERIMENT 1. We implemented our algorith on 50 pencils $(A, B)$ on each different dimension, where $A$ is symmetric tridiagonal random matrix with both diagonal and off-diagonal elements being uniformly distributed random numbers between 0 and 1. $B$ is a symmetric tridiagonal matrix with off-diagonal elements, $\gamma_{i}$, being uniformly distributed random numbers between 0 and 1 , and its diagonal elements $\delta_{i}=2 \max \left(\gamma_{i}, \gamma_{i+1}\right)$. 
The computations were done on a Sun SPARC station 1.

Table 1 shows the results of our algorithm HRST and the algorithm RSG in EISPACK [4]. The algorithm RSG first reduces $A x=\lambda B x$ to $\tilde{A} y=\lambda y$, then solves it. Since $\tilde{A}$ is a full matrix, the RSG is unattractive for this problem as we mentioned in section 1 . But the RSG is the only algorithm available for this problew in EISPACK.

\begin{tabular}{|l|r|r|r|r|r|l|c|c|}
\hline \multirow{2}{*}{$\begin{array}{l}\text { Order } \\
\mathrm{N}\end{array}$} & \multicolumn{2}{|c|}{$\begin{array}{c}\text { Execution Time } \\
\text { all eigenvalues }\end{array}$} & \multicolumn{2}{|c|}{$\begin{array}{c}\text { Execution Time } \\
\text { all eigenpairs }\end{array}$} & \multicolumn{2}{|c|}{$\max _{i}\left\|A x_{i}-\lambda_{i} B x_{i}\right\|_{2} / \lambda_{\max }$} & \multicolumn{2}{|c|}{$\max _{i, j}\left|\left(X^{T} X-I\right)_{i, j}\right|$} \\
\cline { 2 - 9 } & RGS & HRST & RGS & HRST & RGS & HRST & RGS & HRST \\
\hline 60 & 4.25 & 1.9 & 6.9 & 2.1 & $3.16 \mathrm{D}-14$ & $8.32 \mathrm{D}-15$ & $1.19 \mathrm{D}-14$ & $4.91 \mathrm{D}-14$ \\
121 & 35.4 & 5.8 & 69.6 & 7.1 & $3.56 \mathrm{D}-14$ & $1.75 \mathrm{D}-14$ & $1.42 \mathrm{D}-14$ & $1.63 \mathrm{D}-14$ \\
180 & 95.4 & 10.8 & 185.6 & 13.4 & $5.41 \mathrm{D}-14$ & $2.83 \mathrm{D}-15$ & $1.84 \mathrm{D}-14$ & $8.02 \mathrm{D}-14$ \\
241 & 287.4 & 18.7 & 429.4 & 22.4 & $5.57 \mathrm{D}-14$ & $7.10 \mathrm{D}-14$ & $2.66 \mathrm{D}-14$ & $5.73 \mathrm{D}-14$ \\
\hline
\end{tabular}

Table 1:Average execution time (second) of computed eigenvalues and eigenvectors.

\begin{tabular}{|c|c|c|c|c|c|c|}
\hline \multirow{2}{*}{$\begin{array}{r}\text { Matrix } \\
\text { Type }\end{array}$} & \multirow{2}{*}{$\begin{array}{r}\text { Order } \\
\mathrm{N}\end{array}$} & \multicolumn{2}{|c|}{ Execution Time(sec.) } & \multirow{2}{*}{$\begin{array}{c}\text { Ratio } \\
\text { (DSTEBZ)/HRST) }\end{array}$} & \multicolumn{2}{|c|}{$\left(\sum_{i=1}^{n j}(\alpha(i)-\lambda(i))\right) / \lambda_{\max }$} \\
\hline & & HRST & DSTEBZ & & HRST & DSTEBZ \\
\hline \multirow{4}{*}[1,2,1]{} & 65 & 0.76 & 2.42 & 3.18 & - $1.2219 \mathrm{D}-15$ & $-5.3290 D-15$ \\
\hline & 125 & 2.81 & 8.57 & 3.05 & $3.2201 \mathrm{D}-15$ & $1.0658 \mathrm{D}-14$ \\
\hline & 255 & 11.35 & 34.78 & 3.06 & $-8.6600 \mathrm{D}-15$ & $-2.1316 \mathrm{D}-14$ \\
\hline & 499 & 42.50 & 130.21 & 3.06 & $3.8858 \mathrm{D}-15$ & $7.9936 \mathrm{D}-15$ \\
\hline \multirow{4}{*}{ Wilkinso } & 65 & 0.73 & 1.52 & 2.08 & $-2.6906 \mathrm{D}-14$ & $1.1368 \mathrm{D}-13$ \\
\hline & 125 & 1.92 & 5.12 & 2.67 & $-7.2474 \mathrm{D}-15$ & $5.6843 \mathrm{D}-14$ \\
\hline & 255 & 6.70 & 19.34 & 2.89 & $6.6745 \mathrm{D}-16$ & $2.8421 \mathrm{D}-14$ \\
\hline & 499 & 21.68 & 72.45 & 3.34 & $-2.2760 \mathrm{D}-16$ & 2.5871D-15 \\
\hline
\end{tabular}

Table 2: The results of comparison of HRST with DSTEBZ.

EXPERIMENT 2. (a) $A$ is the Toeplitz matrix $[1,2,1]$, i.e., all diagonal elements, $\alpha(i)$, are 2 and off-diagonal elements are $1 . \quad B=I$.

(b) $A$ is the Wilkinson matrix, i.e., the watrix $[1, \alpha(i), 1]$, where $\alpha(i)=$ abs $((n+1) / 2-i), i=1,2, \ldots, n$ with $n$ odd. $B=I$.

Table 2 shows the computational results comparing our algorithm HRST with the bisection algorithm DSTEBZ in LAPACK [1] on these two problens. It appears that our algorithm leads in speed by a considerable margin in comparison with the DSTEBZ.

EXPERIMENT 3. Matrices $A$ and $B$ are obtained from piece wise linear finite element [11] discretization of the Sturm-Liouvill problem

$$
-\frac{d}{d x}\left(p(x) \frac{d u}{d x}\right)+q(x) u=\lambda u
$$

where $u=u(x), 0<x<\pi$ and $u(0)=u^{\prime}(\pi)=0$ and $p(x)>0$. Here, both $A$ and $B$ are symmetric tridiagonal and positive definite. We use $p(x)=1$ and $q(x)=6$.

The computations were executed on BUTTERFLY GP 1000 , a shared memory multiprocessor machine. 
The speed-up is defined as

$$
S_{p}=\frac{\text { execution time using one processor }}{\text { execution time using processors }}
$$

and the efficiency is the ratio of the speed-up cover $p$.

\begin{tabular}{|c|c|c|c|c|c|c|c|c|c|c|c|}
\hline Order & \multicolumn{9}{|c|}{$n=500$} & \multicolumn{6}{c|}{$n=1000$} \\
\hline Nodes & 1 & 4 & 8 & 16 & 32 & 1 & 4 & 8 & 16 & 32 & 64 \\
\hline ExeTime & 368.4 & 96.64 & 53.48 & 30.99 & 18.75 & 1584 & 407.9 & 217.7 & 120.7 & 70.76 & 45.78 \\
\hline$S_{p}$ & 1.0 & 3.81 & 6.89 & 11.9 & 19.7 & 1.0 & 3.88 & 7.28 & 13.1 & 22.4 & 34.6 \\
\hline$S_{p} / p$ & 1.0 & 0.95 & 0.86 & 0.74 & 0.61 & 1.0 & 0.97 & 0.91 & 0.82 & 0.70 & 0.54 \\
\hline
\end{tabular}

Table 3: Execution time(second), speed-up and efficiency of the algorithm HRST.

Table 3 shows the execution time and the speed-up $S_{p}$ as well as the efficiency $S_{p} / p$ of our algorithm. It appears that our algorithm is very efficient. The natural parallelism of our algorithm makes it an excellent candidate for wultiprocessor machines.

ACKNOWLEDGEMENT: The research was supported in part by the Summer Research Grant from the University of West Florida. Author would like to acknowledge the valuable suggestions from discussions with Tien-Yien Li and Zhonggang Zeng.

\section{REFERENCES}

1. ANDERSON, E., et.al., LAPACK User's Gusde, SIAM, Philadelphia, 1992.

2. CHU, M.T., LI, T.Y., and SAUER, T. Homotopy Method for General A-Matrix Problems, SIAM J. Matrix Anal. and Appl., Vol., 9, No. 4, 528-536, (1988).

3. DONGARRA, J.J. and SORENSEN, D.C. A fully parallel algorithm for symmetric eigenvalue problems, SIAM J. Sci. Stat. Comput. Vol. 8 (1987) s139-s154.

4. GARBOW, B.S., et. al. Matrix ergensystem routines-EISPACK Guide Extension, New York, Springer-Verlag (1977).

5. ILSE, I. and JESSUP, E. Solving the symmetric tridiagonal eigenvalue problem on the Hypercube, SIAM J. Sci. Stat. Comput. Vol. 11 (1990), 203-229.

6. LI, K. and LI, T.Y. An Algorithm for Symmetric Tridiagonal Eagen-problems-Divide and Conquer with Homotopy Continuation. To appear on SIAM, J. Sci. Stat. Comput.

7. LI, T.Y. and SAUER, T. Homotopy method for generalized eigenvalue problems, Linear Alg. Appl. 91(1987), 65-74.

8. PARLETT, B.N. The symmetric eigenvalue problem. Prentice-Hall, Inc., Englenwood Cliffs, NJ (1980).

9. PETERS, G. and WILKINSON, J.H. $A x=\lambda B x$ and the generalzed ezgenproblem, SIAM J. Numer. Anal., 7(1970), 479-492.

10. RELLICH, F. Perturbation theory of eigenvalue problems, Gordon and Breach Science Publishers, New York, NY (1969).

11. STRANG, G. and FIX, G. An analysis of the finite element method. Prentice-Hall, Inc., Englenwood $\mathrm{Cl}$ iffs, NJ (1973).

12. WILKINSON, J.H. The algebrasc ezgenvalue problem, New York, Oxford University Press (1965). 


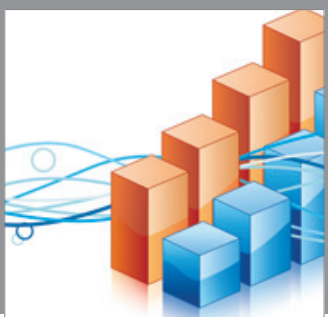

Advances in

Operations Research

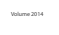

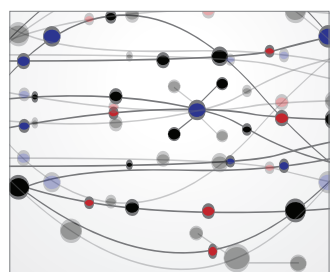

\section{The Scientific} World Journal
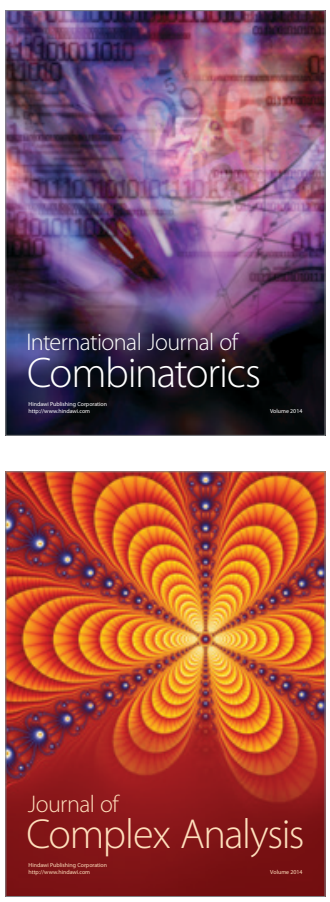

International Journal of

Mathematics and

Mathematical

Sciences
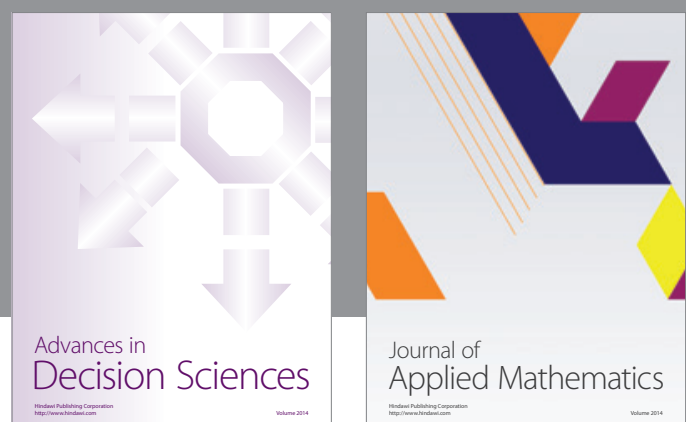

Journal of

Applied Mathematics
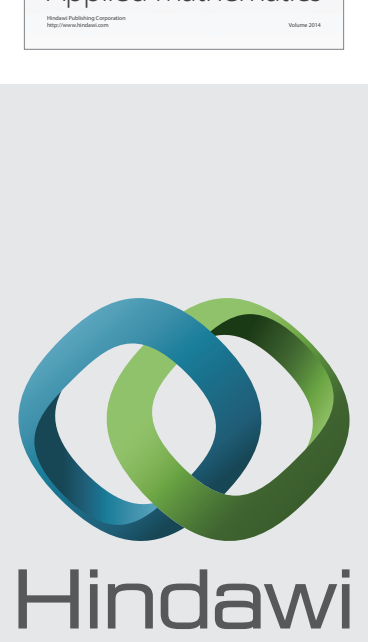

Submit your manuscripts at http://www.hindawi.com
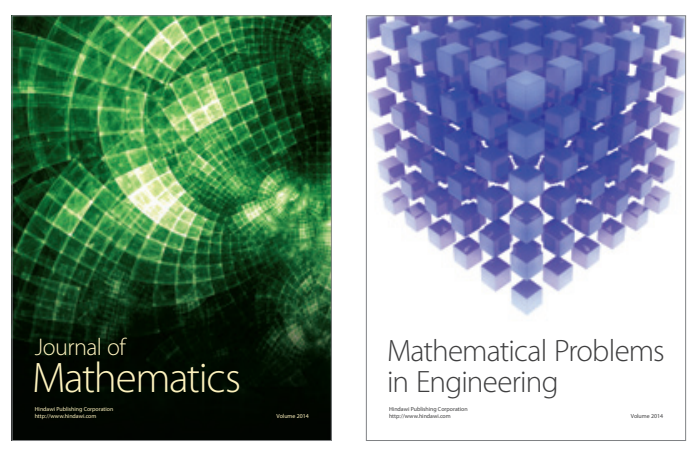

Mathematical Problems in Engineering
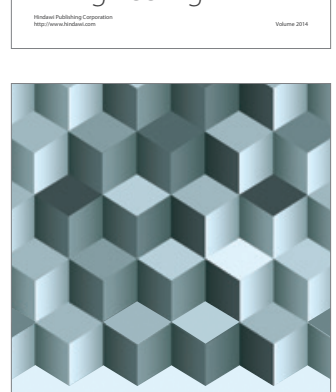

Journal of

Function Spaces
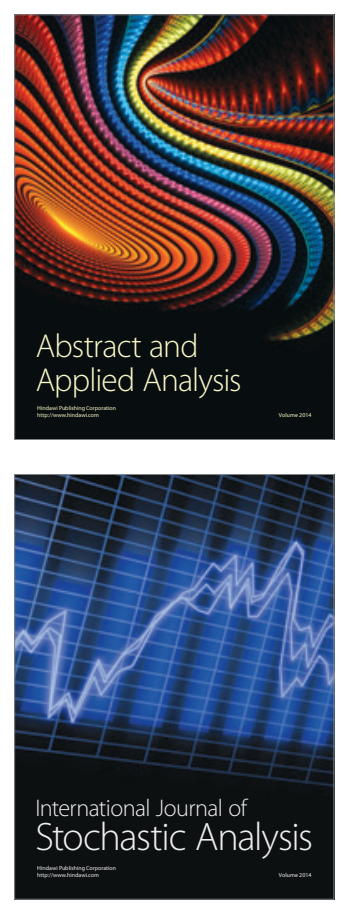

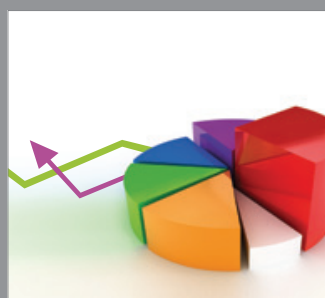

ournal of

Probability and Statistics

Promensencen
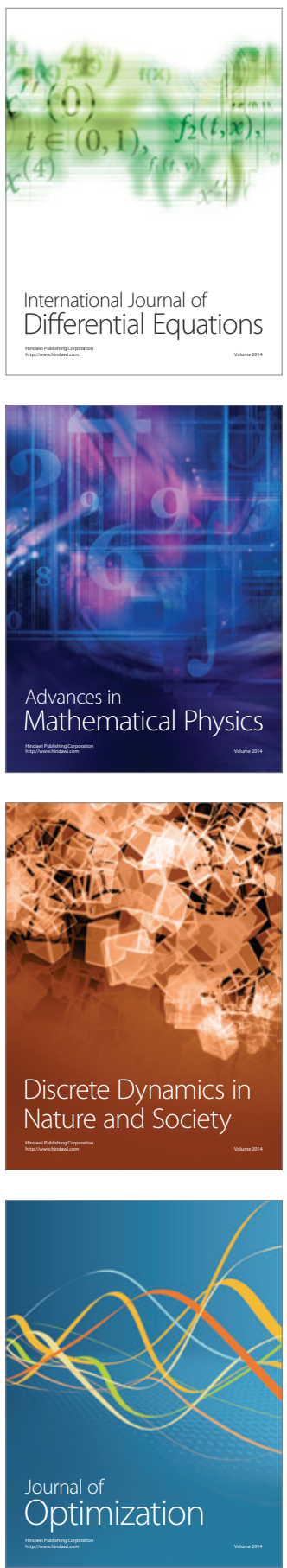